\title{
Les mesures de déformation des structures hyperstatiques: le témoin sonore
}

\author{
Strain measurement on hyperstatic structures : \\ the vibrating wire gauge
}

\author{
A. BOCHON \\ Ingénieur, Direction des Travaux EOLE, SNCF*
}

Rev. Franç. Géotech. n 60 , pp. $41-50$ (juillet 1992)

\section{Résumé}

Les effets des variations de température sur une structure hyperstatique, en contrainte comme en déformation, peuvent être évalués grâce à son " taux de liberté ", rapport de la déformation thermique in situ à la déformation thermique libre. Deux exemples illustrent cette notion.

Le témoin sonore, dont le principe de fonctionnement est expliqué, fournit la déformation réelle. La déformation non thermique peut ensuite être estimée en prenant en compte le taux de liberté mesuré. On aboutit enfin aux variations de contrainte, aussi bien thermiques que rhéologiques.

Cette méthode d'interprétation est appliquée aux mesures effectuées dans le tunnel SNCF de Villejust (ligne TGV Atlantique).

\footnotetext{
Abstract

Thermic variations effects on an hyperstatic structure, in stress and in strain alike, can be estimated by its " rate of freedom ", ratio of in situ thermic strain to free thermic strain. Two examples explain this notion.

The vibrating wire gauge, which working principle is explained, provides the true strain. Then the non thermic strain can be estimated by taking into account the measured rate of freedom. Finally the stress variations, thermic and rheologic as well, can be reached.

This interpretation method is applied to the data obtained in Villejust tunnel (SNCF, high speed railway line TGV Atlantique).
} 


\section{INTRODUCTION}

Tout au long de sa vie, un ouvrage de génie civil conçu comme une structure hyperstatique subit un certain nombre de transformations : des variations de forme et des évolutions de ses contraintes internes. Ces modifications mécaniques représentent une part du phénomène de lente dégradation de l'ouvrage, sans que l'on sache distinguer le facteur le plus nocif entre l'évolution des déformations et la variation des contraintes.

L'instrumentation habituellement mise en place sur les ouvrages d'art permet, grâce à la méthode proposée ici, de connaître à tout moment les déformations et les variations de contraintes en dissociant clairement les effets thermiques des effets rhéologiques.

\section{COMPLÉMENTARITÉ CALCUL-MESURES}

La conception d'un ouvrage donne lieu à des calculs qui permettent de fixer sa forme, ses dimensions et d'évaluer le niveau des sollicitations qu'il aura à subir. Si les actions que doivent être capables de supporter les ouvrages en superstructure sont, dans la plupart des cas, connues et définies par des textes réglementaires, il n'en est pas de même pour les ouvrages souterrains dont le chargement est constitué par le terrain encaissant dont laction est variable à la fois dans l'espace et dans le temps.

Pendant et après la construction de l'ouvrage, son auscultation permet de s'assurer que le mode de travail de la structure, la répartition des déformations et des contraintes, les niveaux de sollicitation sont conformes aux prévisions.

Dans le domaine des ouvrages souterrains plus que dans tout autre, les mesures de déformation et de contrainte sont le moyen de valider les hypothèses et les résultats des calculs, c'est-à-dire de s'assurer que le complexe terrain-ouvrage se comporte comme prévu, et que tous les phénomènes naturels pouvant avoir une influence sur la tenue de la structure ont bien été pris en compte.

\section{HYPERSTATICITÉ, EFFET THERMIQUE ET TAUX DE LIBERTÉ}

Les principales causes de déformation d'un ouvrage sont au nombre de trois ;

- le chargement, avec ses effets immédiat et différé ;

- les variations thermiques, entrainant contractions

et dilatations ;

- les variations hygrométriques, qui provoquent le retrait ou le gonflement du béton.

Dans les structures isostatiques, les contraintes résultent du chargement et, dans une moindre mesure, des phénomènes hydriques qui peuvent induire des hétérogénéités superficielles ou localisées.
Dans les structures hyperstatiques, du fait de la surabondance des liaisons au niveau des appuis, tout facteur de déformation agit également sur le champ de contraintes, mais seuls les effets immédiats du chargement relèvent de la théorie de l'élasticité.

Par exemple, un buton calé à ses deux extrémités contre les parois d'une fouille et exposé aux rayons du soleil, ne se dilate que pour autant que le massif de sol soutenu accepte cette dilatation. $\mathrm{Si}$, comme c'est généralement le cas à court terme, le sol se comporte comme un matériau quasiment élastique, le buton, en se dilatant, comprime le sol à ses deux extrémités et provoque une augmentation des réactions d'appui horizontales, équilibrée par une augmentation proportionnelle de la contrainte de compression du buton.

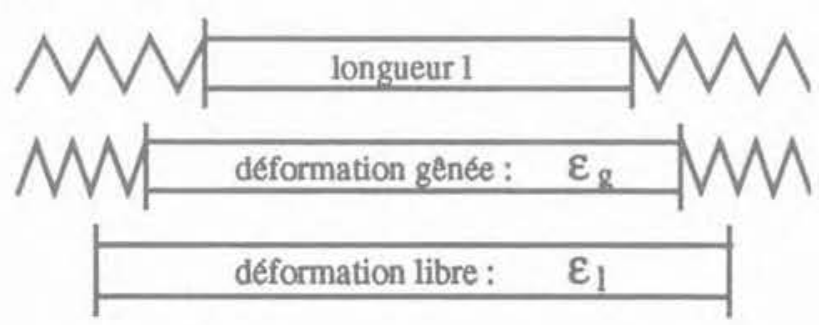

Fig. 1. - Déformations thermiques gênée et libre.

Fig. 1. - Restrained and free thermic strains.

La déformation gênée mesurée est une fraction de la déformation que provoquerait le même écart de température sur la structure en déformation libre.

Le rapport de la déformation thermique gênée à la déformation thermique libre dans la direction envisagée sera appelé taux de liberté de la structure:

$$
\mathrm{n}=\frac{\epsilon_{\mathrm{g}}}{\epsilon_{\mathrm{l}}}
$$

La valeur de $\mathrm{n}$ est comprise entre 0 pour une structure à déformation empêchée et 1 pour une structure à déformation libre (structure isostatique).

La déformation thermique gênée due à un écart de température $\theta$ s'écrit donc :

$$
\epsilon_{\mathrm{g}}=\mathrm{n} \beta \theta
$$

$\beta$ : coefficient de dilatation thermique du matériau constitutif de la structure.

La variation de contrainte concomitante est proportionnelle à la différence entre la déformation thermique réelle $\epsilon_{\mathrm{g}}$ et la déformation thermique libre $\epsilon_{\mathrm{g}}$ :

$$
\sigma=E\left(\epsilon_{\mathrm{g}}-\epsilon_{\mathrm{g}}\right)
$$

d'où :

$$
\sigma=E(\mathrm{n}-1) \beta \theta
$$

Toute variation de température se traduit donc pour une structure hyperstatique par une déformation de même signe accompagnée d'une variation de contrainte de signe opposé :

$$
\epsilon=\mathrm{n} \beta \theta \quad \sigma=\mathrm{E}(\mathrm{n}-1) \beta \theta
$$


Variation de contrainte et déformation sont donc liées par une loi de comportement des structures hyperstatiques linéaires soumises à des variations thermi. ques:

$$
\sigma_{\mathrm{t}}=\mathrm{E}\left(1-\frac{1}{\mathrm{n}}\right) \epsilon_{\mathrm{t}}
$$

Cette loi est applicable tant que $\mathrm{n}$ appartient au domaine semi-ouvert ]0, 1]. Lorsque n est nul (structure bloquée), la variation de contrainte nous est fournie par la formule (2):

$$
\text { si } n=0 \quad \epsilon=0 \quad \sigma=-E \beta \theta
$$

Le taux de liberté $n$ représente donc la sensibilité de la structure aux variations thermiques, sachant qu'un taux de liberté élevé signifie que la structure réagit essentiellement en se déformant, et qu'inversement un taux de liberté faible suppose que les écarts de température se manifestent surtout par des variations de contraintes.

Pour illustrer les notions présentées ci-dessus, nous choisirons tout d'abord un exemple simple mais peu réaliste, celui d'un buton qui travaillerait seul, sans l'intermédiaire d'écrans de soutènement. Nous étudierons ensuite un cas réel, celui d'un revêtement de galerie souterraine.

Premier exemple: calcul du taux de liberté d'un buton.

Il s'agit de comparer le déplacement $\delta 1$ de chaque extrémité du buton avec la variation de sa contrainte interne. Nous pouvons résoudre ce problème linéaire par la formule de Boussinesq donnant l'enfoncement $\delta 1$ dans le sol d'une plaque circulaire en fonction de la force appliquée $\mathrm{P}$ :

$$
\delta 1=\frac{\mathrm{P}}{\mathrm{D}} \frac{1-\nu_{s}^{2}}{\mathrm{E}_{\mathrm{s}}}
$$

$\mathrm{E}_{\mathrm{g}}$ et $\nu_{\mathrm{s}}$ caractérisant le sol. D étant le diamètre de la plaque.

D'où l'expression de la contrainte interne du buton :

$$
\sigma=-\delta \mid \frac{\mathrm{D}}{\mathrm{S}} \frac{\mathrm{E}_{\mathrm{S}}}{1-\nu_{\mathrm{s}}^{2}}
$$

Le signe - est justifié par le fait qu'une variation de température induit une déformation de même signe et une variation de contrainte de signe opposé (voir formule 2).

Par comparaison avec la loi de comportement (3) en posant $\epsilon=2 \delta \mathrm{l} / \mathrm{l}$ on obtient l'expression du taux de liberté du buton:

$$
\frac{1}{n}=1+\frac{1 D}{2 E S} \frac{E_{s}}{1-\nu_{s}^{2}}
$$

Soit un buton constitué d'un profilé HEB 300 de $149 \mathrm{~cm}^{2}$ de section, de $10 \mathrm{~m}$ de longueur, appuyé au sol par des platines de $0,50 \mathrm{~m}$ ou $1 \mathrm{~m}$ de diamètre. Avec $\nu_{s}=0,3$ nous obtenons pour $n$ les valeurs suivantes :

\begin{tabular}{|c|c|r|r|r|}
\hline$E_{\mathrm{s}}(\mathrm{MPa})=$ & \multicolumn{1}{|c|}{100} & \multicolumn{1}{|c|}{500} & \multicolumn{1}{c|}{1000} & \multicolumn{1}{c|}{2500} \\
\hline$D=0,50 \mathrm{~m}$ & 0,92 & 0,69 & 0,53 & 0,31 \\
\hline$D=1 \mathrm{~m}$ & 0,85 & 0,53 & 0,36 & 0,19 \\
\hline
\end{tabular}

Deuxième exemple : calcul du taux de liberté du revêtement d'une galerie circulaire en milieu élastique isotrope.

Les effets d'une variation de tempétature sur le revêtement d'une galerie sont de deux ordres.

déformation : $\quad \frac{\mathrm{u}}{\mathrm{R}}=\mathrm{n} \beta \theta$

variation de contrainte: $\sigma_{\mathrm{b}}=\mathrm{E}_{\mathrm{b}}(\mathrm{n}-1) \beta \theta$

L'équation d'équilibre du complexe terrain-revêtement lie la contrainte tangentielle interne du revêtement à la pression d'extrados:

$$
\sigma_{\mathrm{b}}=\mathrm{p} \frac{\mathrm{R}}{\mathrm{e}}
$$

Si nous prenons pour hypothèse que la température n'agit pas sur le sol, son comportement peut être décrit par les lois de l'élasticité :

$$
\frac{u}{\mathrm{R}}=-\mathrm{p} \frac{1+\nu_{\mathrm{s}}}{\mathrm{E}_{\mathrm{s}}}
$$

$\mathrm{u}$ est la variation du rayon $\mathrm{R}, \mathrm{u} / \mathrm{R}$ est donc de signe opposé à $\mathrm{p}$ (un accroisssement de rayon provoque une compression à l'extrados).

On peut extraire la pression p d'une part des deux équations donnant $\mathrm{u} / \mathrm{R}$ :

$$
\mathrm{p}=-\mathrm{n} \beta \theta \frac{\mathrm{E}_{\mathrm{s}}}{1+\nu_{\mathrm{s}}}
$$

d'autre part des deux équations donnant la contrainte dans le revêtement:

$$
\mathrm{p}=\frac{\mathrm{e}}{\mathrm{R}} \mathrm{E}_{\mathrm{b}}(\mathrm{n}-1) \beta \theta
$$

Ces deux équations nous permettent d'obtenir l'expression du taux de liberté d'un revêtement de galerie :

$$
\frac{1}{n}=1+\frac{R}{e E_{b}} \frac{E_{s}}{1+\nu_{s}}
$$

L'abaque de la page suivante donne la valeur du taux de liberté d'une galerie revêtue en fonction des modules du terrain (en abscisse) et du revêtement (en ordonnée). L'épaisseur du revêtement est égale au dixième du rayon de la galerie, le coefficient de Poisson du sol est égal à 0,3 .

Remarque : il existe en matière de variation de température des cas plus complexes, comme les structures soumises à un gradient thermique, par exemple les poutres sur appuis multiples qui présentent deux taux de liberté différents : l'un proche de l'unité vis-à-vis des variations de température globales et l'autre, beaucoup 


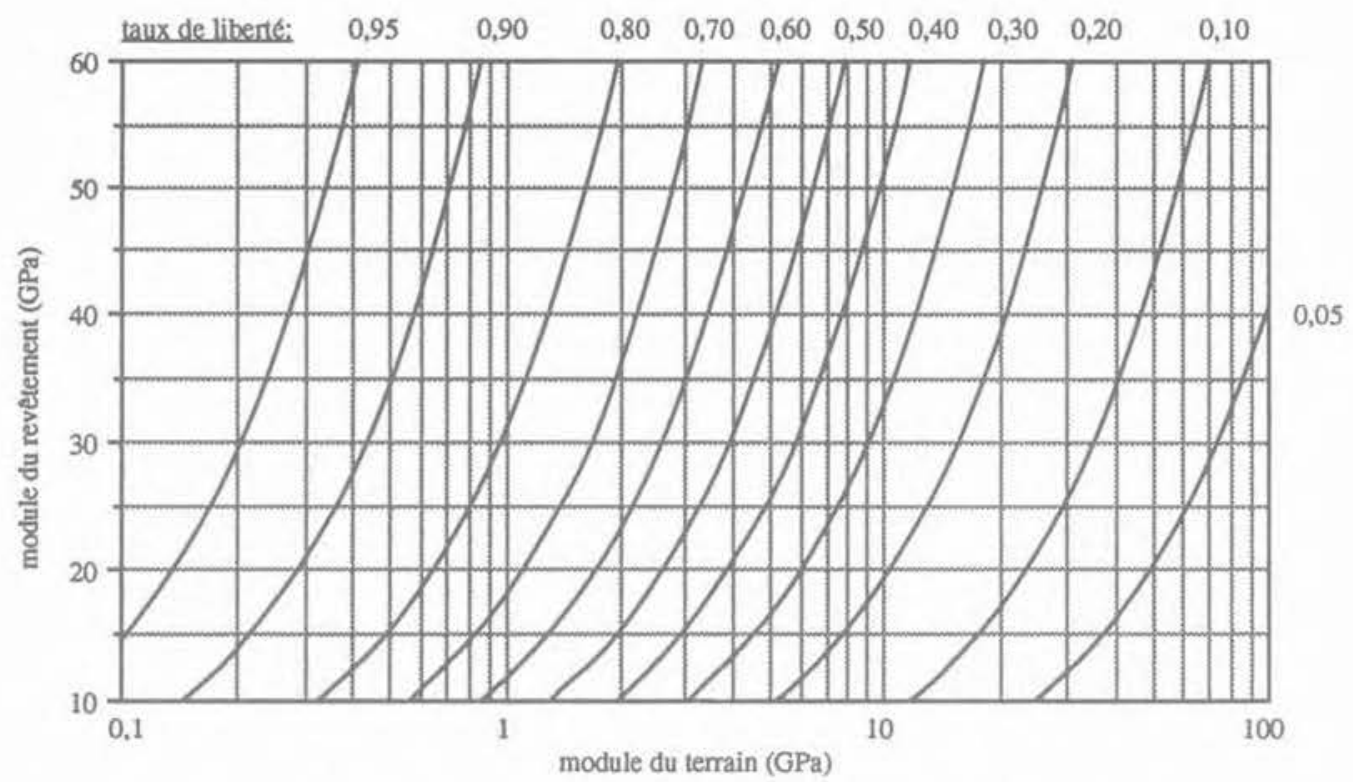

Fig. 2. - Valeur du taux de liberté en fonction des modules du revêtement et du terrain. Fig. 2. - Rate of freedom value versus the lining and soil Young modulus.

plus faible, vis-à-vis de ce gradient thermique. Ce dernier se manifeste par un moment fléchissant :

$$
M=E I(n g r-1) \frac{\beta \Theta g r}{h}
$$

\section{LE TÉMOIN SONORE}

\subsection{Description}

Dans le domaine du génie civil, l'appareil le plus utilisé pour mesurer les déformations et en déduire des contraintes, en particulier à long terme, est le témoin sonore à corde vibrante. Cet appareil est constitué d'un fil d'acier tendu entre deux bases solidaires de la pièce auscultée : l'appareil peut être noyé dans le béton ou fixé sur un profilé métallique ou sur tout autre support. A côté du fil d'acier est disposé un électro-aimant chargé de le faire entrer en vibration,

La conception de ce capteur est telle que sa présence n'a pas d'influence sensible sur le phénomène mesuré (il ne constitue pas une inclusion rigide).

La vibration d'une corde flexible tendue entre deux bases fixes est décrite par l'équation de mouvement :

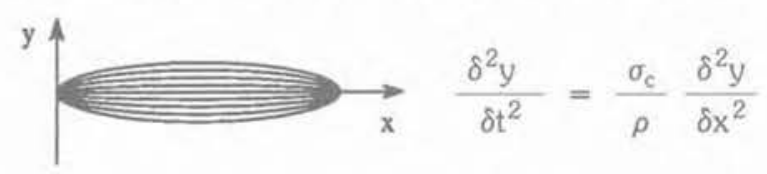

$\rho$ et $\sigma_{\mathrm{c}}$ respectivement masse volumique et contrainte interne de la corde.

Cette équation admet pour solution :

$$
\begin{aligned}
& y=\left[A \sin \left(\frac{\omega}{a} x\right)\right. \\
& \left.+B \cos \left(\frac{\omega}{a} x\right)\right] \sin (\omega t+\Phi)
\end{aligned}
$$

avec :

$$
\mathrm{a}^{2}=\frac{\sigma_{\mathrm{c}}}{\rho}
$$

La fréquence de vibration d'une corde de longueur 1 à extrémités fixes $(y=0$ pour $x=0$ et $x=1)$ est donnée par l'expression :

$$
\mathrm{f}=\frac{\omega}{2 \pi}=\frac{1}{21} \sqrt{\frac{\sigma_{c}}{\rho}}
$$

Le principe de la mesure du témoin sonore consiste à exciter le fil et à mesurer sa fréquence de vibration qui est donc fonction des trois paramètres $1, \sigma_{c}$ et $\rho$. On peut exprimer le carré de la fréquence en fonction de $1, S$ et $\sigma_{c}$ :

$$
f^{2}=\frac{1}{4 m} \frac{S \sigma_{c}}{1}
$$

avec $\mathrm{m}$ la masse de la corde (valeur constante) : $\mathrm{m}=\rho \mathrm{S}$ I et $\mathrm{S}$ section transversale de la corde.

$\mathrm{Si} \mathrm{l}_{0}$ et $\mathrm{S}_{0}$ sont la longueur et la section de la corde au repos, dl et dS étant les déformations provoquées par la mise en tension initiale à la contrainte $\sigma_{\mathrm{c}}$, on peut écrire :

$$
\mathrm{f}^{2}=\frac{1}{4 \mathrm{~m}}\left[\left(\mathrm{~S}_{\mathrm{o}}+\mathrm{dS}\right) \frac{\sigma_{\mathrm{c}}}{\mathrm{l}_{\mathrm{o}}+\mathrm{dl}}\right]
$$

Les variations de longueur et de température agissant d'une part sur $\sigma_{c}$, d'autre part sur dl et dS, I et $S$ apparaissent comme des paramètres de deuxième ordre par rapport à $\sigma_{c}$. Nous pouvons donc négliger leurs variations et considérer $\sigma_{c}$ comme seul paramètre agissant sur la fréquence.

La formule (4) permet d'exprimer la contrainte interne de la corde:

$$
\sigma_{c}=41^{2} \rho f^{2}
$$


et par suite de la remarque ci-dessus :

$$
\sigma_{\mathrm{c}}=4 \rho \mathrm{l}^{2}\left(\mathrm{f}^{2}-\mathrm{f}_{\mathrm{o}}{ }^{2}\right)
$$

D'où, en posant :

$$
\begin{gathered}
k=\frac{4 \rho l^{2}}{E_{c}} \\
\sigma_{c}=E_{c} k\left(f^{2}-f_{o}{ }^{2}\right)
\end{gathered}
$$

Le facteur $k$ est dénommé « coefficient extensométrique ", c'est une caractéristique fournie par le fabricant pour chaque type de corde vibrante.

L'indication de la température étant une donnée importante pour l'interprétation, on mesure également sur les témoins sonores P'évolution de la température à l'endroit du capteur par la variation de la résistance de la bobine.

\subsection{Déformation réelle}

\section{et déformation non thermique}

Une corde vibrante est hyperstatique par rapport à son support car leurs déformations sont égales par principe, mais leur sensibilité thermique généralement différente (la sensibilité thermique du support étant représentée par le produit $n \beta$ défini au paragraphe 2). La déformation gênée de la corde est donc égale à la somme des déformations non thermique et thermique du support :

$$
\epsilon_{\mathrm{g}}=\epsilon_{\mathrm{to}}+\mathrm{n} \beta \theta
$$

La déformation libre de la corde serait uniquement due à la température :

$$
\epsilon_{1}=\alpha \theta
$$

$\alpha$ : coefficient de dilatation thermique de l'acier de la corde.

D'où, en appliquant la formule (1), la contrainte interne de la corde :

$$
\sigma_{c}=E_{c}\left(\epsilon_{\text {to }}+(n \beta-\alpha) \theta\right)
$$

Les formules (5) et (6) permettent maintenant d'exprimer les déformations en fonction des variations de fréquence :

$$
\epsilon_{\mathrm{to}}+(\mathrm{n} \beta-\alpha) \theta=\mathrm{k}\left(\mathrm{f}^{2}-\mathrm{f}_{\mathrm{o}}{ }^{2}\right)
$$

Cette expression permet de calculer d'une part la déformation :

$$
\epsilon=\epsilon_{\mathrm{to}}+\mathrm{n} \beta \theta
$$

qui est donc la déformation réelle :

$$
\epsilon=k\left(f^{2}-f_{0}{ }^{2}\right)+\alpha \theta
$$

et d'autre part la déformation à température cons. tante :

$$
\epsilon_{\mathrm{to}}=\mathrm{k}\left(\mathrm{f}^{2}-\mathrm{f}_{\mathrm{o}}{ }^{2}\right)+(\alpha-\mathrm{n} \beta) \theta
$$

Si les conditions de mesure sont telles qu'il est permis de supposer les déformations non thermiques nul- les $\left(\epsilon_{\text {to }}=0\right)$, on peut calculer le terme $n \beta$ à partir de l'expression (9), les autres termes étant soit mesurés $\left(\mathrm{f}, \mathrm{f}_{\mathrm{o}}, \Theta\right)$ soit caractéristiques de la corde $(\mathrm{k}, \alpha)$ :

$$
n \beta=\frac{k\left(f^{2}-f_{o}{ }^{2}\right)}{\theta}+\alpha
$$

On peut ensuite introduire cette valeur dans l'expression (9) pour calculer les déformations non thermiques.

\section{Remarque sur la notion d'auto-compensation}

Le principe de lauto-compensation du témoin sonore veut que la fréquence de vibration de la corde ne soit pas affectée par les variations de température. Pour cela, le corps de l'appareil est constitué d'un tube en acier, dont le coefficient de dilatation thermique est le même que celui de la corde.

Dans le cas où le témoin sonore est laissé sans support ou si il est fixé sur un support isostatique en acier, on a $\mathrm{n}=1$ et $\beta=\alpha$ et la formule (6) montre que la contrainte thermique de la corde est nulle. La température n'agit donc pas sur la fréquence de vibration.

Si maintenant le témoin sonore est fixé sur un support tel que $\beta$ soit différent de $\alpha$ ou $n$ différent de 1 , alors la température influe sur la contrainte interne de la corde et, par conséquent, sur sa fréquence de vibration.

Il apparaît ainsi que dans la plupart des configurations réelles, à savoir structures isostatiques non métalliques et structures hyperstatiques, l'auto-compensation n'existe pas.

\subsection{Aspect pratique}

- Le coefficient $n \beta$, représentant la déformabilité thermique de la structure, peut être évalué en procédant à une série de mesures pendant une période suffisamment brève pour que les déformations non thermiques soient négligeables $\left(\epsilon_{t_{0}} \approx 0\right) ; n \beta$ est alors la pente de la droite représentant la fonction $\epsilon=f(\theta)$. Il est important que ce coefficient soit mesuré sur chaque témoin sonore car les conditions d'appui, donc le taux de liberté $n$, dépendent de la direction de la déformation mesurée et dans certains cas de sa localisation dans la structure.

- La déformation non thermique $\epsilon_{t o}$ se décompose généralement en trois termes :

- $\epsilon_{\mathrm{e}}$ déformation élastique instantanée sous contrainte,

et deux termes non élastiques :

- $\epsilon_{\mathrm{f}}$ représentant le fluage du matériau, qui n'induit pas de contraintes ;

- $\epsilon_{\mathrm{h}}$ représentant les phénomènes hydriques des matériaux poreux comme le gonflement et le retrait du béton.

$$
\epsilon_{\mathrm{to}}=\epsilon_{\mathrm{e}}+\epsilon_{\mathrm{i}}+\epsilon_{\mathrm{h}}
$$

Remarque: la valeur $\epsilon_{\text {to }}$ représente la déformation non thermique de la structure, mais cette déformation peut tout de même avoir une origine thermique, ce serait par exemple le cas du revêtement d'une galerie souterraine soumise à des variations de température importantes (stockage de gaz liquéfié) et par 
conséquent aux variations de volume d'une couronne de terrain encaissant. Les valeurs de $\epsilon_{10}$ mesurées sur le revêtement traduiraient alors l'effet mécanique sur celui-ci des déformations thermiques du terrain.

- La déformation de fluage $\epsilon_{j}$ peut être évaluée par le calcul ou mesurée grâce à des essais spécifiques (chargements d'éprouvettes sur bâtis de fluage).

- Les déformations hydriques $\epsilon_{h}$ peuvent être évaluées par calcul ou, ce qui est nettement prétérable, mesurées à l'aide de blocs témoins isolés en contraintes et intégrés à la structure pour être soumis aux mêmes conditions hygrométriques.

- Le coefficient de dilatation thermique $\beta$ du matériau peut être mesuré sur ces mêmes blocs témoins, leur taux de liberté $n$ étant égal à 1 du fait qu'ils sont isolés en contraintes du reste de la structure.

- Le taux de liberté $n$ peut être calculé comme le rapport du terme $n \beta$ mesuré sur la structure au coefficient $\beta$ mesuré sur les blocs témoins.

Dès lors que l'on dispose de valeurs pour les termes $\epsilon_{f}$ et $\epsilon_{h}$, il devient possible de calculer la déformation élastique sous contrainte $\epsilon_{\mathrm{e}}$, en notant tout de même qu'elle peut représenter l'effet d'un état de contraintes bi ou tridimensionnel, auquel cas il est intéressant de disposer de mesures de déformation dans deux ou dans les trois directions principales.

Les influences respectives des déformations cyclique et acyclique peuvent également être approchées grâce à la " méthode saisonnière globale " développée par EDF pour l'auscultation des grands barrages.

\subsection{Evaluation des contraintes}

Pour simplifier l'exposé, nous ferons l'hypothèse d'un champ de contraintes uniaxial.

Nous avons vu plus haut que dans une structure hyperstatique les contraintes ont deux origines.

Tout d'abord le chargement, qui induit un champ de contraintes dont nous mesurons l'effet en déformation. L'interprétation demande donc de connaitre le module d'Young et le coefficient de Poisson du matériau. Ces caractéristiques peuvent être connues (structures métalliques), mais dans le cas du béton il est nécessaire de mesurer au moins le module d'Young soit par des essais sur éprouvettes, soit sur la structure elle-même par mesure des déformations sous un chargement connu et de courte durée (épreuves d'ouvrage de franchissement, poussée de tunnelier sur un revêtement de galerie...). L'expression de la déformation élastique sous contrainte $\epsilon_{e}$ est déduite de la formule (10) :

$$
\epsilon_{e}=\epsilon_{\mathrm{to}}-\epsilon_{\mathrm{f}}-\epsilon_{\mathrm{h}}
$$

En multipliant cette déformation par le module d'élasticité instantané du matériau, la déformation de fluage étant déjà prise en compte $\left(\epsilon_{f}\right)$ et en remplaçant $\epsilon_{\text {to }}$ par sa valeur (formule 9), on obtient l'expression de la contrainte correspondant à la déformation élastique :

$$
\sigma_{e}=E_{1}\left[k\left(f^{2}-f_{0}{ }^{2}\right)+(\alpha-n \beta) \theta-\epsilon_{f}-\epsilon_{h}\right]
$$

Le second facteur de variation des contraintes est l'évolution des températures (cf. paragraphe 2). La formule (2) nous donne la contrainte thermique :

$$
\sigma_{\theta}=E_{1}(n-1) \beta \theta
$$

En sommant ces deux termes, on obtient l'expression de la contrainte totale:

$$
\sigma=E_{i}\left[k\left(f^{2}-f o^{2}\right)+(\alpha-\beta) \theta-\epsilon_{f}-\epsilon_{h}\right]
$$

Remarque : l'interprétation la plus courante des résultats de témoins sonores consiste à utiliser une méthode basées sur les formules:

$$
\begin{aligned}
& \epsilon^{\prime}=k\left(f^{2}-f_{0}{ }^{2}\right)+(\alpha-\beta) \theta \\
& \sigma^{\prime}=E\left(\epsilon^{\prime}-\epsilon_{1}-\epsilon_{h}\right)
\end{aligned}
$$

sensées fournir déformation et contrainte non thermiques.

Cette méthode présente une série d'inconvénients: - elle est basée sur l'hypothèse implicite d'isostaticité de la structure et utilise les lois de l'élasticité, les résultats sont donc faux si cette hypothèse n'est pas vérifiée :

- elle occulte complètement la déformation réelle et la notion de taux de liberté ;

- il est généralement admis que $\sigma$ est la contrainte non thermique alors qu'il s'agit de la contrainte totale (voir formule 11).

Cette méthode d'interprétation ne permet donc, dans le cas d'une structure hyperstatique, qu'une description partielle du comportement de louvrage, laspect thermique lui échappant complètement. Même en cas d'isostaticité elle ne permet qu'une correction thermique approchée car la valeur de $\beta$ est fixée a priori ou, dans le meilleur des cas, mesurée sur éprouvettes.

On peut illustrer les lacunes de la méthode simplifiée par linterprétation des résultats de mesures suivants :

$$
k\left(\mathrm{f}^{2}-\mathrm{fo}^{2}\right)=+9 \quad \theta=-6^{\circ} \mathrm{C}
$$

En tenant compte d'un coefficient de dilatation thermique du béton égal à $10 \mu \mathrm{m} / \mathrm{m} /{ }^{\circ} \mathrm{C}$, valeur souvent adoptée a priori, la méthode simplifiée estime la déformation et la contrainte à :

$$
\epsilon^{\prime}=\sigma^{\prime}=0
$$

les résultats sont en fait les suivants :

- déformation totale : $\epsilon=-60 \mu \mathrm{m} / \mathrm{m}$

- déformation non thermique : $\epsilon_{\mathrm{ro}}=-15 \mu \mathrm{m} / \mathrm{m}$

- contrainte (si $\mathrm{E}=20000 \mathrm{MPa}): \sigma=-0,3 \mathrm{MPa}$.

\subsection{Précision des résultats}

Le témoin sonore, comme tout appareil de mesures, ne fournit des résultats précis et fiables que sous certaines conditions qui concernent essentiellement le respect de la procédure de mise en œuvre préconisée par le fabricant. Il convient ensuite de s'assurer que le capteur, une fois en place dans la structure, four- 
nira bien la mesure attendue, ce qui suppose le respect de l'implantation et de l'orientation prévues.

Il importe de noter que la grandeur mesurée par ce type de capteur est une fréquence de vibration, fonction de deux variables, la déformation et la température du support, ayant des influences comparables sur le résultat de la mesure.

Si l'interprétation de ces résultats en termes de déformation ne fait appel qu'aux grandeurs mesurées, il n'en est pas de même pour la recherche des contraintes qui intègre, cornme nous l'avons vu plus haut, des caractéristiques issues de calculs théoriques ou d'essais de laboratoire (fluage, phénomènes hydriques, module d'Young, coefficient de Poisson). L'évaluation des erreurs proposée ici porte uniquement sur les résultats en déformation, la précision des valeurs des caractéristiques physiques du matériau étant fonction de leur mode d'évaluation.

Nous prendrons en compte les précisions suivantes : - $\mathrm{d}\left[\mathrm{k}\left(\mathrm{f}^{2}-\mathrm{f}_{0}{ }^{2}\right)\right]=0,002$ de l'étendue de mesure, soit en moyenne $4 \mu \mathrm{m} / \mathrm{m}$ pour une étendue de mesure de $2000 \mu \mathrm{m} / \mathrm{m}$;

$-\mathrm{d} \theta=0,5^{\circ} \mathrm{C}$

$-\alpha=11,5 \mu \mathrm{m} / \mathrm{m} /{ }^{\circ} \mathrm{C}, \mathrm{d} \alpha / \alpha=1 \%$.

Soient les deux relevés de mesure suivants :

$$
\begin{array}{ll}
1-k\left(f^{2}-f^{\circ}{ }^{2}\right)=-104 & \theta=10^{\circ} \mathrm{C} \\
2-k\left(f^{2}-f_{0}^{2}\right)=-139 & \theta=4{ }^{\circ} \mathrm{C}
\end{array}
$$

Calculons l'erreur sur la déformation réelle $\epsilon$,

- valeur de la déformation:

$$
\epsilon=k\left(f^{2}-f_{o}{ }^{2}\right)+\alpha \theta
$$

- erreur sur la déformation :

$$
\begin{aligned}
\mathrm{d} \epsilon=\mathrm{d}[\mathrm{k} & \left.\left(\mathrm{f}^{2}-\mathrm{fo}^{2}\right)\right]+\alpha \theta\left(\frac{\mathrm{d} \alpha}{\alpha}+\frac{\mathrm{d} \theta}{\theta}\right) \\
\epsilon_{1} & =11 \mu \mathrm{m} / \mathrm{m} \pm 11 \mu \mathrm{m} / \mathrm{m} \\
\epsilon_{2} & =-93 \mu \mathrm{m} / \mathrm{m} \pm 10 \mu \mathrm{m} / \mathrm{m}
\end{aligned}
$$

Supposons que le coefficient de dilatation thermique $\mathrm{n} \beta$ soit évalué à $9,5 \pm 1 \mu \mathrm{m} / \mathrm{m} /{ }^{\circ} \mathrm{C}$ par une série de mesures spécifiques.

Calculons maintenant l'erreur sur la déformation à température constante $\epsilon_{\text {to }}$,

- valeur de la déformation:

$$
\epsilon_{\mathrm{to}}=\epsilon-\mathrm{n} \beta \Theta
$$

- erreur sur la déformation :

$$
\begin{aligned}
& \mathrm{d} \epsilon_{\mathrm{to}}=\mathrm{d} \epsilon+(\mathrm{n} \beta \theta)\left(\frac{\mathrm{dn} \beta}{\mathrm{n} \beta}+\frac{\mathrm{d} \theta}{\theta}\right) \\
& \epsilon_{\mathrm{to1}}=-84 \mu \mathrm{m} / \mathrm{m} \pm 26 \mu \mathrm{m} / \mathrm{m} \\
& \epsilon_{\mathrm{to} 2}=-131 \mu \mathrm{m} / \mathrm{m} \pm 19 \mu \mathrm{m} / \mathrm{m}
\end{aligned}
$$

L'erreur sur la déformation réelle est de l'ordre de la dizaine de microns, mais l'erreur sur la déformation à température constante est 2,5 fois plus importante ; celle-ci induit donc lors de linterprétation en contrainte une erreur égale à $0,5 \mathrm{MPa}$ en supposant un module de $20000 \mathrm{MPa}$.

\section{Remarques}

- L'erreur la plus importante est due à la mesure de température, la précision des résultats pourrait être améliorée par la mise en place de sondes thermiques permettant une mesure à $0,1^{\circ} \mathrm{C}$ près. Les valeurs ci-dessus se présenteraient alors de la manière suivante :

$$
\begin{gathered}
\epsilon_{1}=11 \mu \mathrm{m} / \mathrm{m} \pm 6 \mu \mathrm{m} / \mathrm{m} \\
\epsilon_{\mathrm{to} 1}=-84 \mu \mathrm{m} / \mathrm{m} \pm 17 \mu \mathrm{m} \\
\epsilon_{2}=-93 \mu \mathrm{m} / \mathrm{m} \pm 6 \mu \mathrm{m} / \mathrm{m} \\
\epsilon_{\mathrm{to} 2}=-131 \mu \mathrm{m} / \mathrm{m} \pm 11 \mu \mathrm{m} / \mathrm{m}
\end{gathered}
$$

L'erreur sur les déformations serait dans ces conditions deux fois plus faible.

- La bonne connaissance du comportement d'un ouvrage nécessite la mise en place de sections de mesures équipées chacune d'un certain nombre de témoins sonores. Or la précision est améliorée par le nombre de mesures de la même grandeur: en matière de mesures de déformation, la redondance sert la précision. Par exemple, s'il est possible d'appliquer à chacune des sections de mesure d'une poutre lhypothèse de NAVIER-BERNOUILLI sur les sections planes, on peut réduire l'erreur sur les mesures de déformation réelle en rapportant les résultats à un plan de déformation moyen.

\section{EXEMPLE DE CALCUL DE DÉFORMATIONS ET D'ÉVALUATION DE CONTRAINTES : \\ LES TUNNELS DE VILLEJUST}

L'ouvrage de Villejust, situé sur la ligne TGV Atlantique, est constitué de deux tunnels parallèles de $4800 \mathrm{~m}$ de longueur et de $8,24 \mathrm{~m}$ de diamètre intérieur, creusés au tunnelier à boue bentonitique et revêtus d'anneaux de voussoirs préfabriqués de $40 \mathrm{~cm}$

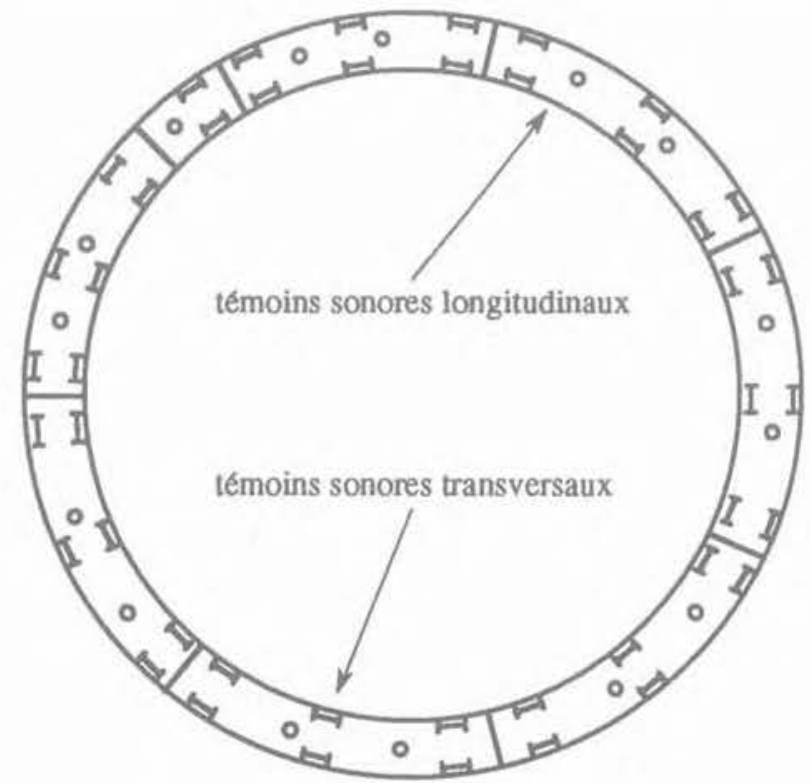

Fig. 3. - Implantation des térnoins sonores dans un anneau de voussoirs.

Fig. 3. - Vibrating wire gauges position in a lining ring. 
d'épaisseur. Quatre anneaux de voussoirs d'un tunnel ont été équipés chacun de 74 témoins sonores : 30 pour mesurer les déformations longitudinales et 44 par paires pour mesurer les déformations tangentielles à l'intrados et à P'extrados.

Afin de simplifier le développement, la méthode est appliquée aux valeurs moyennes relevées sur un anneau (anneau $n^{\circ} 31$, voie 2 , tête Sud).

\subsection{Visualisation des déformations réelles}

Le graphe ci-dessous représente les déformations réelles moyennes dans le sens tangentiel (extrados et intrados) et dans le sens longitudinal. On a superposé à ce graphe la courbe des températures avec une échelle telle qu'une variation de $1^{\circ} \mathrm{C}$ a la même amplitude qu'une déformation de $10 \mu \mathrm{m} / \mathrm{m}$.

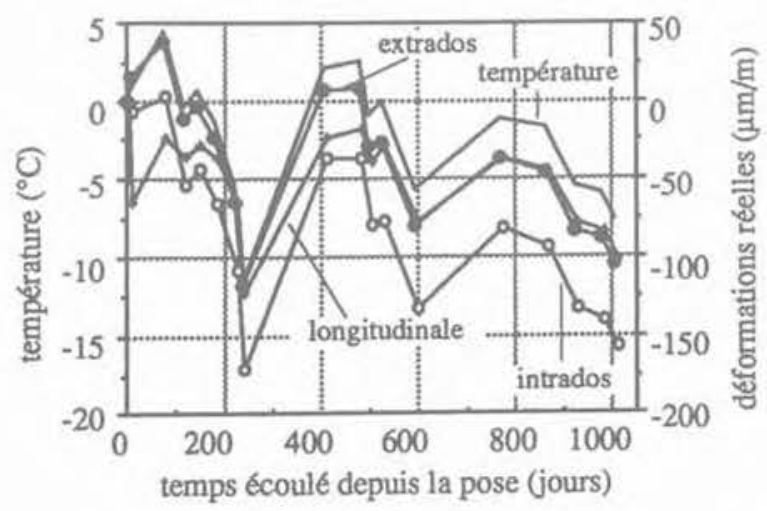

Fig. 4. - Evolution à long terme des déformations réelles et de la température de l'anneau.

Fig. 4. - Long term evolution of true strains and of the temperature of the ring.

Les courbes de température et de déformation tangentielle sont presque parallèles, ce qui permet de supposer un coefficient de dilatation thermique $n \beta$ voi- sin de $10 \mu \mathrm{m} / \mathrm{m} /{ }^{\circ} \mathrm{C}$ dans le sens transversal. Par contre, l'amplitude des déformations longitudinales est plus faible, d'où probablement un coefficient plus réduit dans ce sens.

Le graphique fait apparaître un important différentiel de déformation entre l'extrados et l'intrados qui peut être expliqué par un phénomène déjà observé dans quelques tunnels : après mise en place du revêtement, l'extrados n'est plus aéré alors que l'intrados l'est toujours, quelquefois avec une hygrométrie plus faible que celle qu'a connu le revêtement préfabriqué à lextérieur du tunnel. On observe donc la poursuite du phénomène de retrait à l'intrados, voire son accélération, alors que l'extrados n'évolue plus et peut même subir un certain gonflement au contact de la nappe phréatique ou en cas d'injection du vide annulaire avec un coulis très liquide.

\subsection{Recherche des coefficients de dilatation thermique}

La représentation des déformations réelles en fonction de la température permet de tracer des droites de régression dont les pentes sont égales aux coefficients de dilatation thermique recherchés (fig. 5).

Ces valeurs de $n \beta$ calculées sur les voussoirs confirment les observations faites sur le graphe des déformations réelles.

Le bloc témoin fait apparaître le coefficient $\beta$ de dilatation thermique du matériau (fig. 6).

\subsection{Calcul des taux de liberté}

En considérant un coefficient de dilatation thermique du béton égal à $11,1 \mu \mathrm{m} / \mathrm{m} /{ }^{\circ} \mathrm{C}$ (valeur mesurée sur le bloc témoin), nous obtenons trois taux de liberté différents :

$\mathrm{n}=0,95$ pour l'extrados ;

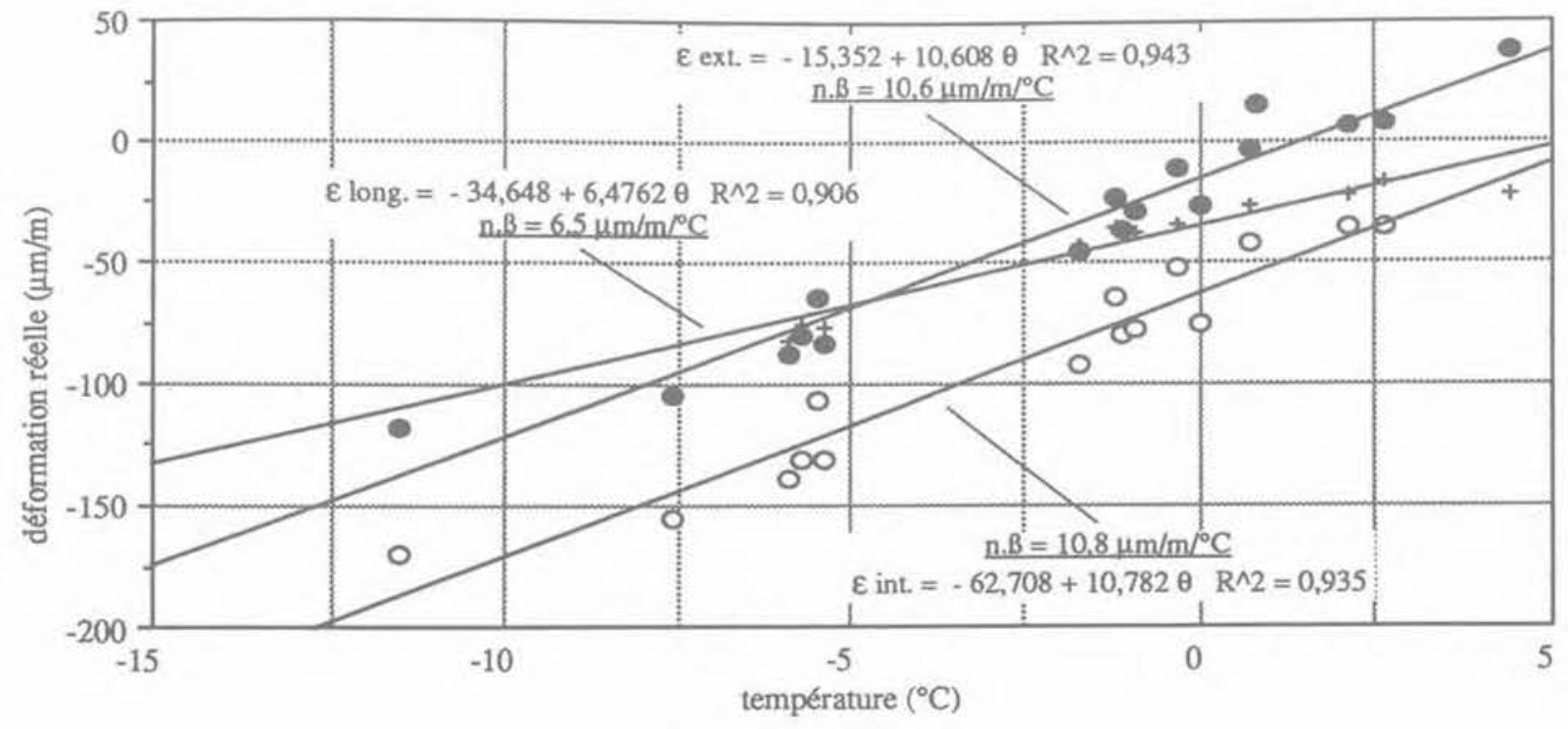

Fig. 5. - Déformations réelles de l'anneau en fonction de la température.

Fig. 5. - True strains of the ring versus temperature. 


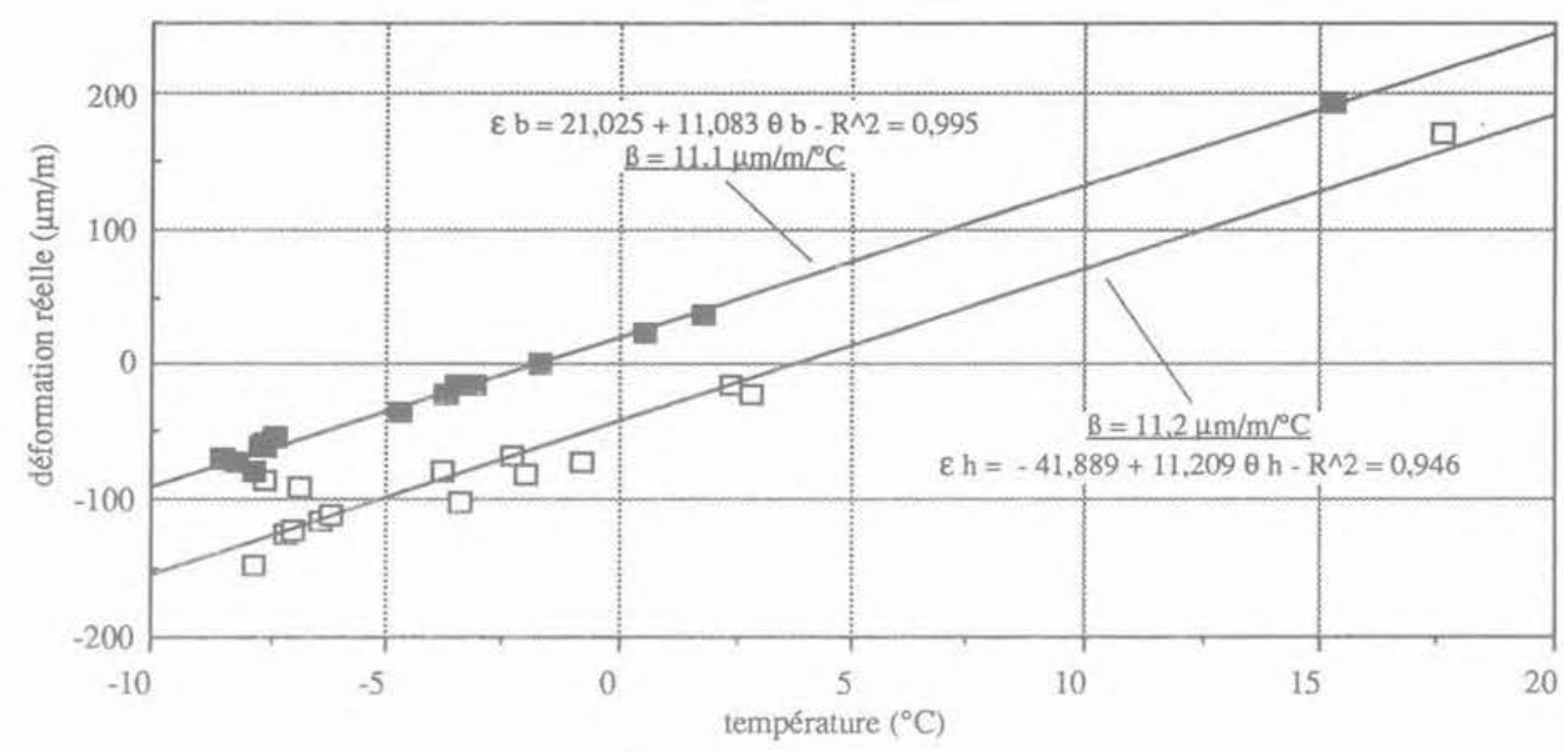

Fig. 6. - Déformations réelles du bloc témoin en fonction de la température. Fig 6 , - True strains of the check sample versus temperature.

$\mathrm{n}=0,97$ pour l'intrados ;

$\mathrm{n}=0,59$ dans le sens longitudinal.

Cette dernière valeur, que l'on aurait pu attendre égale à 0 du fait de la continuité du revêtement et en supposant un blocage parfait des anneaux entre eux, s'explique par une certaine latitude en déformation tolérée par les joints boulonnés garnis de feutre bitumineux.

La petite différence entre les valeurs d'intrados et d'extrados est peut-être à relier aux phénomènes hydriques évoqués plus haut : l'intrados des voussoirs s'étant rétréci, les joints à faces planes assurent le contact par l'extrados, laissant ainsi l'intrados relativement plus libre vis-à-vis de l'évolution de la température.

\subsection{Calcul des déformations non thermiques}

Les graphiques ci-après représentent l'évolution des déformations non thermiques de l'anneau, c'est-à-dire la superposition des effets du chargement (effet mécanique et fluage) et des variations hydriques. Ces déformations sont calculées par application de la formule (8) avec les coefficients de dilatation thermique déterminés ci-dessus.

Sur la figure 7 (évolution des déformations non thermiques à court terme) l'alternance des phases de foration et de pose apparait clairement:

- sur la courbe des déformations longitudinales, les pointes vers le bas représentent les phases de foration, les vérins du tunnelier exerçant leur poussée sur le revêtement,

- sur les courbes des déformations tangentielles, les variations sont de sens contraire et plus réduites.

Connaissant l'amplitude des déformations $\epsilon_{\mid}$et $\epsilon_{t}$ ainsi que les pressions $\sigma_{1}$ appliquées longitudinalement par les vérins de poussée du tunnelier, il est possible de calculer le module de Young du béton et la contrainte

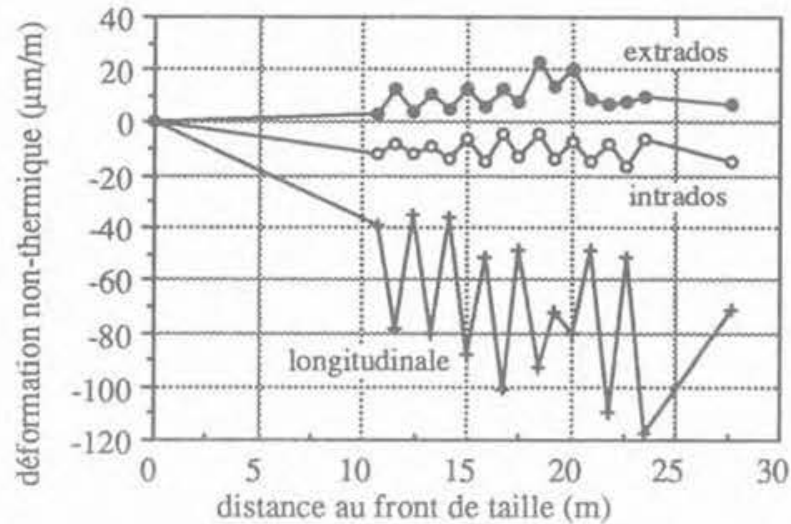

Fig. 7. - Evolution à court terme des déformations réelles de l'anneau en fonction de la distance au front de taille.

Fig. 7. - Short term evolution of true strains of the ring versus the distance to the face.

tangentielle $\sigma_{t}$ dans le revêtement sous la forme d'un problème en contraintes planes, la contrainte radiale $\sigma_{\mathrm{r}}$ pouvant être négligée car beaucoup plus faible que $\sigma_{t}$ et $\sigma_{1}$.

On aboutit à la formulation suivante :

$$
\begin{gathered}
E=\frac{\left(1-\nu^{2}\right) \sigma_{\mathrm{l}}}{\epsilon_{\mathrm{l}}+\nu \epsilon_{\mathrm{t}}} \\
\sigma_{\mathrm{t}}=\left(\epsilon \mathrm{t}+\nu \epsilon_{\mathrm{l}}\right) \frac{\mathrm{E}}{1-\nu^{2}}
\end{gathered}
$$

En prenant pour $\nu$ la valeur 0,2 le module de Young ressort à $45400 \mathrm{MPa}$, à comparer à la valeur moyenne $46700 \mathrm{MPa}$ mesurée sur éprouvettes.

La contrainte tangentielle moyenne dans lanneau atteint $1,4 \mathrm{MPa}$ une semaine après la pose de l'anneau alors que le front de taille s'est déplacé de plus de $25 \mathrm{~m}$ (la déformation de fluage étant prise égale à $20 \%$ de la déformation instantanée). 
On observe d'autre part sur ces courbes :

- une contraction de l'intrados de l'anneau, qui représente probablement le début de la mise en charge du revêtement par le terrain ;

- une dilatation de l'extrados (déformation positive) qui peut s'expliquer par le contact avec un coulis d'injection très liquide.

L'évolution à long terme des déformations non thermiques (fig. 8) confirme le différentiel intradosextrados déjà constaté à court terme et fait apparaître dans le sens longitudinal une détente puis une stabilisation après quatre mois environ.

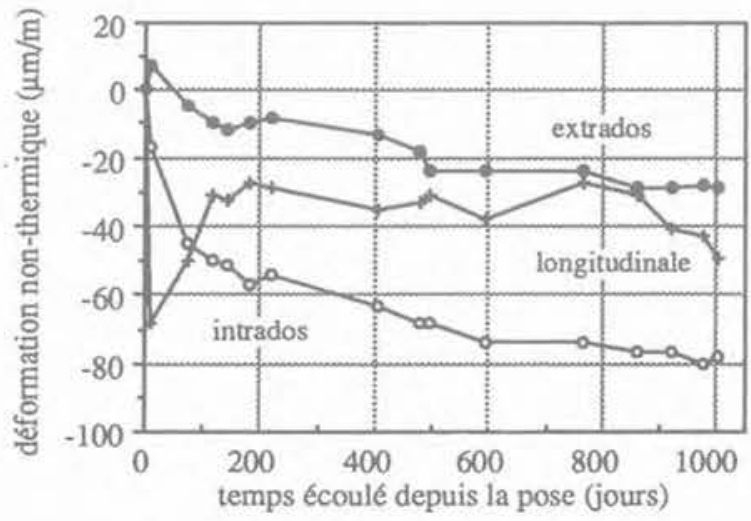

Fig. 8. - Evolution a long terme

des déformations non thermiques de l'anneau.

Fig. 8. - Long term evolution

of the non thermic strains of the ring

Si la courbe d'extrados, en principe non affectée par le retrait, fait apparaître une déformation différée importante, la prise en compte d'un fluage théorique à 1000 jours égal à $85 \%$ de la déformation instantanée suppose une contrainte tangentielle moyenne de 1,5 $\mathrm{MPa}$, soit un accroissement de $0,1 \mathrm{MPa}$ seule. ment sur le long terme.

\section{RÉSUMÉ DE LA MÉTHODE}

5.1. Calcul des déformations réelles:

$$
\epsilon=\mathrm{k}\left(\mathrm{f}^{2}-\mathrm{f}_{\mathrm{o}}{ }^{2}\right)+\alpha \theta
$$

5.2. Calcul du coefficient $\mathrm{n} \beta$ (ou $\beta$ ) pour chaque témoin sonore :

$$
\mathrm{n} \beta=\frac{\mathrm{k}\left(\mathrm{f}^{2}-\mathrm{f}_{\mathrm{o}}{ }^{2}\right)}{\theta}+\alpha
$$

$\mathrm{n} \beta$ est la pente de la droite représentative de la fonction $\epsilon=f(\theta)$ si on ne retient que les mesures satisfaisant au critère $\epsilon_{\text {to }} \approx 0$ (déformations essentiellement thermiques)

le coefficient $\beta$ est fourni par les mesures sur blocs témoins ou éventuellement sur témoins sonores spécifiques pour un champ de contraintes unidimensionnel (il faut dans ce cas tenir compte de l'effet de déformation transversale).

5.3. Calcul du taux de liberté de la structure :

$$
\mathrm{n}=\frac{\mathrm{n} \beta}{\beta}
$$

En théorie on peut caractériser une structure par trois taux de liberté correspondant aux trois directions principales.

5.4. Calcul des déforations non thermiques :

$$
\epsilon_{\mathrm{to}}=\mathrm{k}\left(\mathrm{f}^{2}-\mathrm{f}_{\mathrm{o}}{ }^{2}\right)+(\alpha-\mathrm{n} \beta) \theta
$$

5.5. Recherche des valeurs des déformations différées (fluage et phénomènes hydriques) par calcul ou essais spécifiques :

$$
\epsilon_{\mathrm{f}} \quad \epsilon_{\mathrm{h}}
$$

5.6. Calcul des déformations élastiques :

$$
\epsilon_{e}=\epsilon_{\text {to }}-\epsilon_{\mathrm{i}}-\epsilon_{\mathrm{h}}
$$

5.7. Calcul des contraintes correspondant aux déformations élastiques à l'aide de la loi de Hooke généralisée et autant d'hypothèses simplificatrices que nécessaires :

$$
\left[\sigma_{e}\right]=[\mathrm{C}] \cdot\left[\epsilon_{e}\right]
$$

[C] : matrice d'élasticité du matériau faisant appel au module de Young $E_{1}$ et au coefficient de Poisson $\nu$.

5.8. Calcul des contraintes thermiques :

$$
\sigma_{\Theta}=E_{i}(n-1) \beta \theta
$$

5.9. Calcul des contraintes totales :

$$
\sigma=\sigma_{\mathrm{e}}+\sigma_{\theta}
$$

\section{CONCLUSION}

La méthode proposée ici enrichit l'interprétation des mesures de témoins sonores car elle permet de calculer les déformations réelles. Il est ainsi possible de mesurer sur l'ouvrage lui-même son coefficient de dilatation thermique, et de tenir compte d'un terme correctif précis pour le calcul des déformations non thermiques.

Dans le cas des structures hyperstatiques, ce terme correctif revêt une importance particulière car les écarts de température peuvent être un facteur prépondérant de variation du champs de contraintes. Ce terme fait appel au coefficient de dilatation thermique in situ de la structure, qui n'est qu'une fraction du coefficient de dilatation thermique du matériau qui la constitue, les conditions d’appui réduisant ses possibilités de déformation. Le rapport de ces deux coefficients est appelé « taux de liberté * de la structure.

Cette méthode permet donc de caractériser le type de réponse de l'ouvrage aux variations de température et d'évaluer les contraintes en les dissociant selon leur origine: mécanique ou thermique.

\section{BIBLIOGRAPHIE}

BOCHON A., Mémoire de synthèse des mesures effectuées pendant la construction des tunnels de Villejust. Mémoire d'ingénieur. Conservatoire National des Art et Métiers. Paris 1990.

BONVALET $\mathrm{CH}$., Difficultés d'interprétation des mesures extensométriques à long terme dans les matériaux poreux tels que les bétons et les roches. Revue pratique de contrôle industriel $n^{\circ} 129$. 